Volume 7, No.2, April - June 2018

International Journal of Computing, Communications and Networking

Available Online at http://www.warse.org/ijccn/static/pdf/file/ijccn31722018.pdf

https://doi.org/10.30534/ijccn/2018/31722018

\title{
AUTOMATED SYSTEM FOR AIR POLLUTION DETECTION AND MONITORING IN VEHICLES
}

\author{
Prof.Anusha K L ${ }^{1}$, Lakshmi S R $\mathbf{R}^{2}$, Sowjanya $K^{3}$, Shreya V $S^{4}$, Renuka $\mathbf{N}^{5}$ \\ ${ }^{1}$ Assistant professor of EWIT,INDIA, anushakl.19@gmail.com \\ ${ }^{2}$ Student of EWIT, INDIA, lashmisr1996@ gmail.com \\ ${ }^{3}$ Student of EWIT, INDIA, sowjanyak322@ gmail.com \\ 4 Student of EWIT, INDIA, shreyavs19@gmail.com \\ ${ }^{5}$ Student of EWIT, INDIA, renulathananjuda@gmail.com
}

\begin{abstract}
The main source of atmosphere pollution happens due to vehicles. The high inflow of vehicles in urban areas causing more air pollution and decreasing air quality that leads to severe health diseases. The main objective of the paper is to introduce vehicular pollution monitoring system using Internet of Things (IoT) which can smoothly monitor vehicles violating the emission standard. The proposed systems also assure the existence of wireless sensors for vehicle pollution system that specialize in a straight forward accessibility of real time data through internet using IoT. The measured data is also shared to vehicle owner, traffic department. This system is a low cost and provides good results in controlling the air pollution especially in the urban areas.
\end{abstract}

Keywords: Internet of Things, Radio Frequency Identification, GSM Technology, Gas sensors.

\section{INTRODUCTION}

The main source of pollution in cities is due to vehicles. The increase use of vehicles in cities results in vital increase in the emission load of various toxins into air[1]. As a result, increase in environmental problems which will affect the human health in urban places. Air pollutants from taxies, cars and buses result in the damage of ground level ozone and other respiratory problem like asthma attacks. Transportation is main source for generating carbon monoxide that contributes $72 \%$ of total pollution in the metropolitan cities like Calcutta, Mumbai, Bangalore, and Delhi.

At present, the Indian pollution control board has made the fitness certificate as compulsory for public and commercial vehicles once in a year to control the pollution. Pollution Under Control (PUC) certificate for every six months is mandatory for all group vehicles from the date of registration.

IoT is become helpful in cities for monitoring air pollution from vehicles and also data related to the amount of pollution on different roads of a city can be gathered and analyzed. Recent approaches in sensing technology, especially in the area of

Wireless Sensor Networks (WSNs), it now empowers environmental monitoring in real time at special and temporal scales [2].
This paper specially designed to operate the system using sensor network and gather the information about pollutant levels discharged by the vehicles. IoT is a new technology which draws the consideration for both academia and industry.

[18] IoT is realized as a network of things, each of which can be label using unique ID and convey based on standard communication protocols. IoT accord objects to communicate with one other, to approach information on the web, to store and collect data, and to collaborate with users, thereby creating smart, ubiquitous and perpetually connected environment. To achieve such intelligence within the environments, big technological innovations methods and developments are needed. The researchers sense that it will be potential to detect a newly built shape to IoT, collect with the crack of pervasive devices in the future. The view of IoT is that of everyday life such as vehicles, roadways in public transport systems, wireless pill-shaped cameras in the system of digestive tracks for healthcare applications, air conditioner, or other household things can be attached with sensors, used to track data regarding these things. IoT has a unique addressable things and their virtual illustration on an internet like structure. Such things can add to data about them, or can send real-time sensor information about their state or other properties combined with the things. The unique address things are connected to the web, and the data can send using the protocol that communicates computers to the internet. Since the things can sense the environment and communicate and may generally enable automatic reply to challenging scheme without human interference[17]. The more numbers of objects meanwhile produce information from the environment in an automatic way and enable common and ubiquitous computing.

This paper proposed an embedded system using wireless sensor network that provides a framework for collecting the sensor data at anyplace using IoT [3]. Wireless sensor along with active RFID is used in the proposed wireless sensor system to monitor the vehicular pollution based on IoT.

The paper is organized into five sections. The design and development of the proposed monitoring system is explained in section II. 


\section{EXISTING SYSTEM}

With the increasing number of automobiles, especially in some metropolis, such as Bombay, Bangalore, Delhi it is very impending to resolve the problem of air pollution resulting from automobile exhaust gas [4]. In present situation, air pollution has reached levels judged as hazardous to human health. To fight this problem, the motor emissions standards have been established and promoted in many developed countries for many years.

Furthermore, researchers have also developed some improved measures in vehicle engines or the quality of gasoline. However, these methods seem not to solve radically the emissions pollution problems [5]. The motor emissions standard is very difficult to carry out in real- life.

Although government forces all cars for testing or examining periodically as the local standard, the actual vehicle on-road emissions are usually much higher than those are, which are measured during the emission inspections.

\section{PROPOSED SYSTEM}

(a) Data collection.

The Data Collection module is placed in car. The sensor is placed near the vehicle exhaust. This sensor gives emission values in analog signal in form of volts, which can be digitized using an inbuilt ADC in a microcontroller. These data along with some additional information such as time and vehicle id can be transmitted. A RF transmitter module helps in transmitting to the central node.

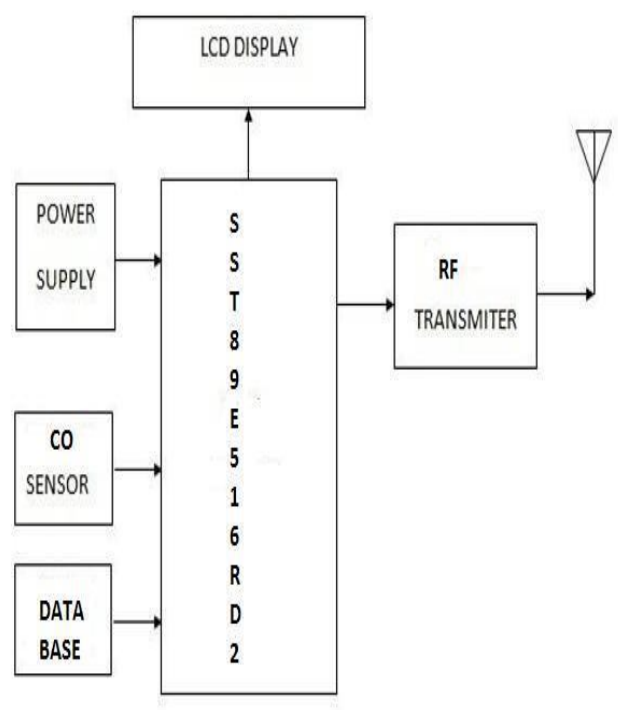

Fig-1: Block diagram of Data collection system (in car).

\section{(b) Data transmission.}

The nodes is installed on pole in city center. This node consists of RF receiver, a microcontroller and GSM module. We can deploy these nodes on many poles in city. Whenever the vehicle moves in range of the RF of node, it receives the transmitted information from the vehicle. We can display the received information on the LCD display at node. Meanwhile the information can be transmitted to the storage system using GSM.

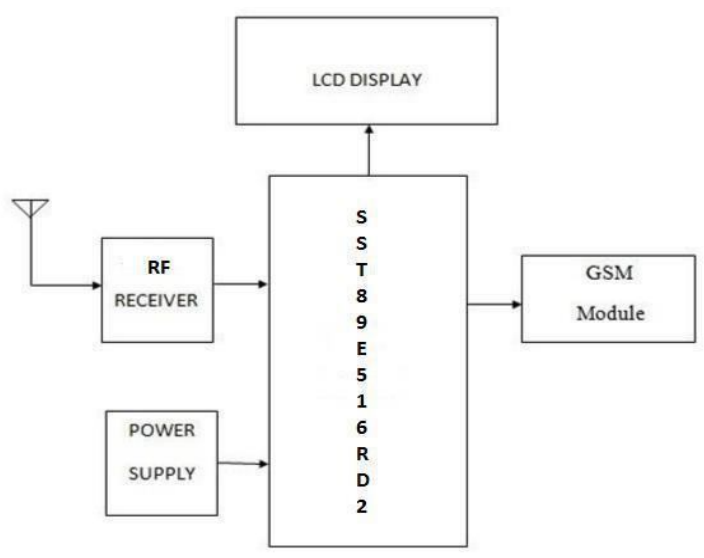

Fig -2: Block diagram of node (at pole)

\section{(c) Notification system}

This system receives the emission values from the node, stores in its database. It compares the emission values with the local emission standard. If the received vehicle emission exceeds the allowed figure, then it will send an alert message to the car owner to repair their cars as soon as possible. Meanwhile, the authorities also can check the car's detail emissions information on a web page. 
Anusha K L et al., International Journal of Computing, Communications and Networking, 7(2) April - June 2018, 174-177

\subsection{FLOW CHART}

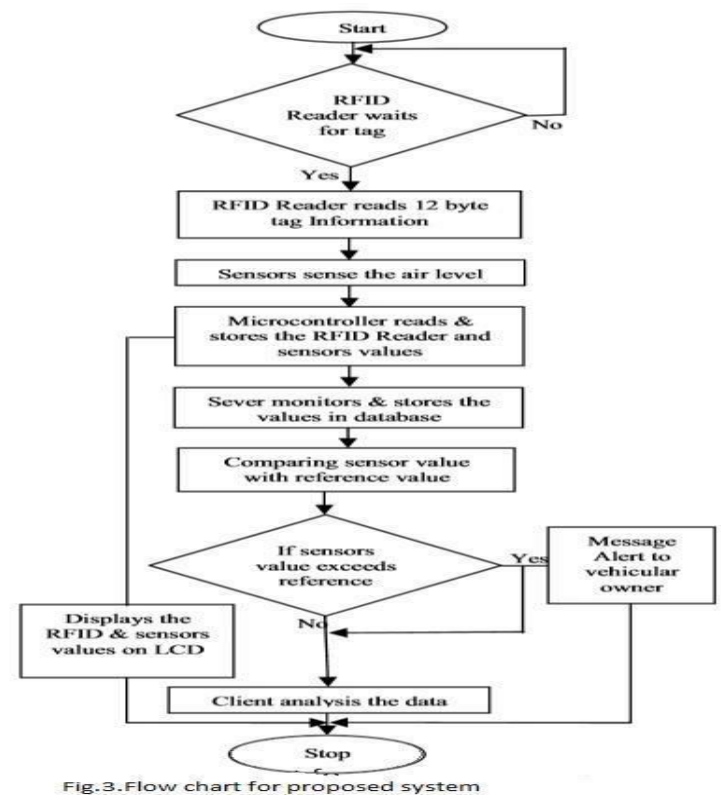

\section{EXPERIMENTS AND RESULTS}

The concentration of gases is directly proportional to output voltage of sensors. The sensor gives an analog voltage varying from $0 \mathrm{~V}$ to $5 \mathrm{~V}$ depending upon concentration of gases. This analog voltage is given to the Analog to Digital converter to get in digital values denoted by V1. After getting V1, the system will proceed with $\mathrm{CO}$ and $\mathrm{HC}$ concentration calculation. The value of $\mathrm{CO}$ concentration is obtained by calculating the value of the sensor resistance (Rs). The value of Rs is obtained from the following equation:

$$
\mathrm{S}=(5-1) / 1
$$

From the log-log graph shown in fig -5 , the relationship between Rs and $\mathrm{CO}$ concentrations in ppm is obtained. Equation 2 shows the relationship between Rs and the CO concentration value. When $\mathrm{x}$-axis equals zero, the intersection value between the graph linear line and the $y$ axis equals 100 . Alpha $(\alpha)$ is the graph slope value.

$$
=100 \times(\mathrm{s}) 1 / \alpha \text { in } p p m--------
$$

By taking two points (first point at $100 \mathrm{ppm}$ and second point at $1000 \mathrm{ppm}$ ) from the linear line graph, the slope can be calculated as

$$
\begin{aligned}
& =(100-1000) /(1-0.1) \\
& =-1
\end{aligned}
$$

With the slope value equals -1 , equation 2 can be simplified into

$$
=100 / \mathrm{s}----------
$$

This equation (5) used to find the CO level in ppm.

Similarly, for MQ-5 sensor, the curve shown in fig -6, meets the $\mathrm{Y}$ axis at Rs/R0 $=5$, and slope of the curve can be founded by eq. (3) which gives $=-0.3927$. The concentration of $\mathrm{HC}$ in $\mathrm{ppm}$ is given by

$$
\begin{aligned}
& =5 /(\text { ) } 2.52 \text {------- } \\
& \text { Conversion of ppm to percentage }(\%) \\
& \mathrm{C}(\%)=\mathrm{C}(\mathrm{ppm}) / 10000
\end{aligned}
$$

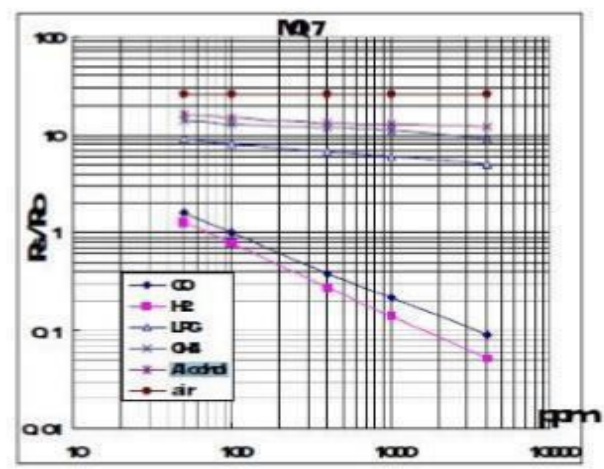

Fig.4. : Graph Sensor resistance ratio (Rs/Ro) versus CO Concentration

\section{CONCLUSION}

This system is cost effective solution for vehicle emission problem. The sensors are used to collect pollutants from vehicle A RF tag is used to collect information regarding emission and then this information transferred to RF receiver which is situated at the signal from where it sends information to server and then the values of emission compared and message sends to vehicle owner. The owner of vehicle has to repair the vehicle within next three message else fine to be paid will be sent in next message and vehicle number will send to respected Control Transportation Authority. The developed system provides better accuracy with low cost.

\section{REFERENCES}

[1] "Epidemiological Study on Effect of Air Pollution On Human Health in Delhi", Environmental Health Management Series: EHMS/0112012, Central Pollution Control Board, Government of India. 
Anusha K L et al., International Journal of Computing, Communications and Networking, 7(2) April - June 2018, 174-177

[2] Z. J. Andersen, M. hyidberg, S. S. Jenson, M. Ketzal, S .Loft. M. Soresen, O. Raashou-Nielsen, "Chronic Obstructive pulmonary disease and long-term exposure to traffic-related air pollution: a cohort," American journal of respiratory and critical caremedicine, 2011, Vol.183 (4), 455-461.

https://doi.org/10.1164/rccm.201006-0937OC

[3] O. Raaschou-Nielsen, Z. 1. Andersen, M. Hvidberg, S. S. Jensen, M. Ketzel, M. Sorensen, ATjonneland, "Lung cancer incidence and long term exposure to air pollution from traffic," Environmental health perspectives, 2011, Vol. 119(6), 860-865.

https://doi.org/10.1289/ehp.1002353

[4] D.L. Yang, F. Liu, and Y.D. Liang, "A survey of the internet of things," Proceedings of International Conference on E-Business Intelligence (ICEBI-2010), Advances in Intelligent Systems Research. Atlantis Press, 2010, pp.358-366.

https://doi.org/10.2991/icebi.2010.72

[5] T. N. Hubbard, "Using inspection and maintenance programs to regulate vehicle emissions", Contemporary Economic Policy, vol. 15, pp. 5262, 2014.

[6] C. K. Chan and X. Yao, "Air pollution in mega cities in China," Atmospheric Environment, vol. 42, pp. 1-42, 2008. tps://doi.org/10.1016/j.atmosenv.2007.09.003

[7] J. H. Visser and R. E. Soltis, "Automotive exhaust gas sensing systems, "IEEE Transactions on Instrumentation and Measurement, vol. 50, pp.1543-1550, Dec 2001.

https://doi.org/10.1109/19.982942

[8] Andrew N Sloss, Dominic Symes, Cris Wright “ARM system developer's guide designing and optimizing system software"2004.

[9] Ya-lin Miaoi", Xiang-lin Miao, Zheng-Zhong Bian, Yongjie Zhang Xi'an Jiaotong University, Xi'an 710049, China "Design and application of Embedded System based on ARM7 LPC2148” Proceedings of the 2005 IEEE.

[10] Srinivasa, R.V, Nageswara, R and Kumari, E.K. (2009). Cloud computing: An overview. Journal of Theoretical and Applied Information Technology, 9(1), 71-76.

[11] ] J. Yang, C. Zhang, X. Li, Y. Huang, S. Fu, M.F. Acevedo. "Integration of wireless sensor networks in environmental monitoring cyber infrastructure", Wireless

Networks, Springer/ACM, Volume 16, Issue 4, pp. 10911108, May 2010 [

[12] Nikhade, Sudhir G.; Agashe, A.A., "Wireless sensor network communication terminal based on embedded Linux and Xbee," Circuit, Power and Computing

Technologies (ICCPCT), 2014

[13] Sheikh Ferdoush, Xinrong Li “Wireless Sensor Network System Design using Raspberry Pi and Arduino for

Environmental Monitoring Applications", Elsevier The 9th International Conference on Future Networks and Communications (FNC-2014)

https://doi.org/10.1016/j.procs.2014.07.059

[14] Jin-Shyan Lee, Yu-Wei Su, and Chung-Chou Shen “ $A$

Comparative Study of Wireless Protocols: Bluetooth, UWB, ZigBee, and Wi-Fi" The 33rd Annual Conference of the IEEE Industrial Electronics Society (IECON) Nov. 5-8, 2007, Taipei, Taiwan

[15] Xiaohui Cheng; Fanfan Shen, "Design of the wireless sensor network communication terminal based on embedded Linux," Software Engineering and Service Science (ICSESS), 2011 IEEE 2nd International Conference on, vol., no., pp.598, 601, 15-17 July 2011

[16] ] J. Yang, C. Zhang, X. Li, Y. Huang, S. Fu, M.F. Acevedo. "Integration of wireless sensor networks in environmental monitoring cyber infrastructure”, Wireless Networks, Springer/ACM, Volume 16, Issue 4, pp. 10911108, May 2010

[17] D.P. Chock, S.L. Winkler, T.Y. Chang, S.J. Rudy, and Z.K. Shen, "Urban ozone air quality impact of emissions from vehicles using reformulated gasolines and M85”, Atmospheric Environment, Vol. 28, pp. 2777-2787, September 2012. https://doi.org/10.1016/1352-2310(94)90081-7

[18] F. Moreno, M. Muñoz, J. Arroyo, O. Magén, and C. Monné, I. Suelves, "Efficiency and emissions in a vehicle spark ignition engine fuelled with hydrogen and methane blends", International Journal of Hydrogen Energy, Vol. 37, Issue 15, pp. 11495-11503, August 2010.

[19] L. Yan, Y. Zhang, L. T. Yang, and H. Ning, "The Internet of things: from RFID to the next-generation pervasive networked systems", Auerbach Publications, 2015 